\title{
The wMelPop strain of Wolbachia interferes with dopamine levels in Aedes aegypti
}

\author{
Luciano A Moreira ${ }^{1,2}$, Yixin H Ye ${ }^{1}$, Karly Turner ${ }^{3}$, Darryl W Eyles ${ }^{3}$, Elizabeth A McGraw ${ }^{1}$, Scott L O'Neill ${ }^{1 *}$
}

\begin{abstract}
Wolbachia is an intracellular bacterium that has been stably transinfected into the mosquito vector of dengue, Aedes aegypti. This inherited infection causes a range of metabolic and phenotypic alterations in the mosquito, which might be related to neuronal abnormalities. In order to determine if these alterations were caused by the manipulation of neuroamines by this bacterium, we studied the expression of genes involved in the dopamine biosynthetic pathway and also measured the amount of dopamine in infected and uninfected mosquitoes of different ages. Wolbachia-infected mosquitoes exhibit greater expression of some genes related to the melanization pathway, but not for those directly linked to dopamine production. Although dopamine levels were higher in Wolbachia-positive mosquitoes this was not consistent across all insect ages nor was it related to the previously described Wolbachia induced "bendy" and "shaky" phenotypes.
\end{abstract}

\section{Findings}

Aedes aegypti is the main vector of dengue, one of the leading arboviral diseases of humans throughout the tropics of the world [1]. In an attempt to develop a new biocontrol approach to reduce dengue transmission, $A$. aegypti mosquitoes have been transinfected with the common inherited bacterial symbiont of insects, Wolbachia [2]. The wMelPop-CLA strain of Wolbachia when present in $A$. aegypti induces a range of effects including reductions in adult lifespan and blockage of vector competence for a range of human pathogens [2-5]. In addition some other effects have also been reported. Infected mosquitoes exhibit increases in both locomotor activity and metabolic rate [6]. As Wolbachia-infected $A$. aegypti females age, they obtain fewer and smaller blood meals and show increasing difficulty in completing the process of blood feeding $[7,8]$. Old female mosquitoes (>15 days) also display behavioral defects described as a "bendy" proboscis or jittering of the body termed "shaky", suggesting that their neuronal function is somehow impaired.

The biogenic amines dopamine, serotonin and octopamine act as signaling molecules in many diverse physiological contexts, including behavior, fertility, and

\footnotetext{
* Correspondence: scott.oneill@uq.edu.au

'School of Biological Sciences, The University of Queensland, Brisbane Qld 4072, Australia

Full list of author information is available at the end of the article
}

reproduction, and in the development of neuronal and non-neuronal tissues [9]. In insects, dopamine is involved in several other biological processes, that span from cuticle formation [10], to egg chorion hardening [11], to gonadotropic regulation [12] and to the immune response [13,14]. Dopamine is associated with locomotor defects in Drosophila [15] and mammals [16] and, in humans its deficiency is the cause of motor defects such as Parkinson disease [17].

Many genes participate in the dopamine biosynthesis pathway, which is highly conserved among vertebrates and invertebrates. Initially, the enzyme tyrosine hydroxylase $(\mathrm{TH})$ [or phenoloxidase - $(\mathrm{PO})$ ] catalyzes the conversion of tyrosine to L-DOPA $[14,18]$, which is then decarboxylated by the enzyme dopa-decarboxylase (DDC) to dopamine (DA) [9] (Figure 1A). In Drosophila, the Ebony protein, which is required for normal behavioral rhythmicity [19] has also been shown to control (through a $\beta$-alanyl-dopamine synthase activity) the dopamine pathway [20].

To examine a possible relationship between dopamine production and the previously described behavioral defects in mosquitoes associated with Wolbachia infection, we characterized transcription of a number of genes involved in the dopamine biosynthetic pathway [21] (Figure 1A) in mosquito heads at 3 different adult ages. A. aegypti mosquitoes, wMelPop-CLA infected and tetracycline cured strains [2], were reared in a controlled 


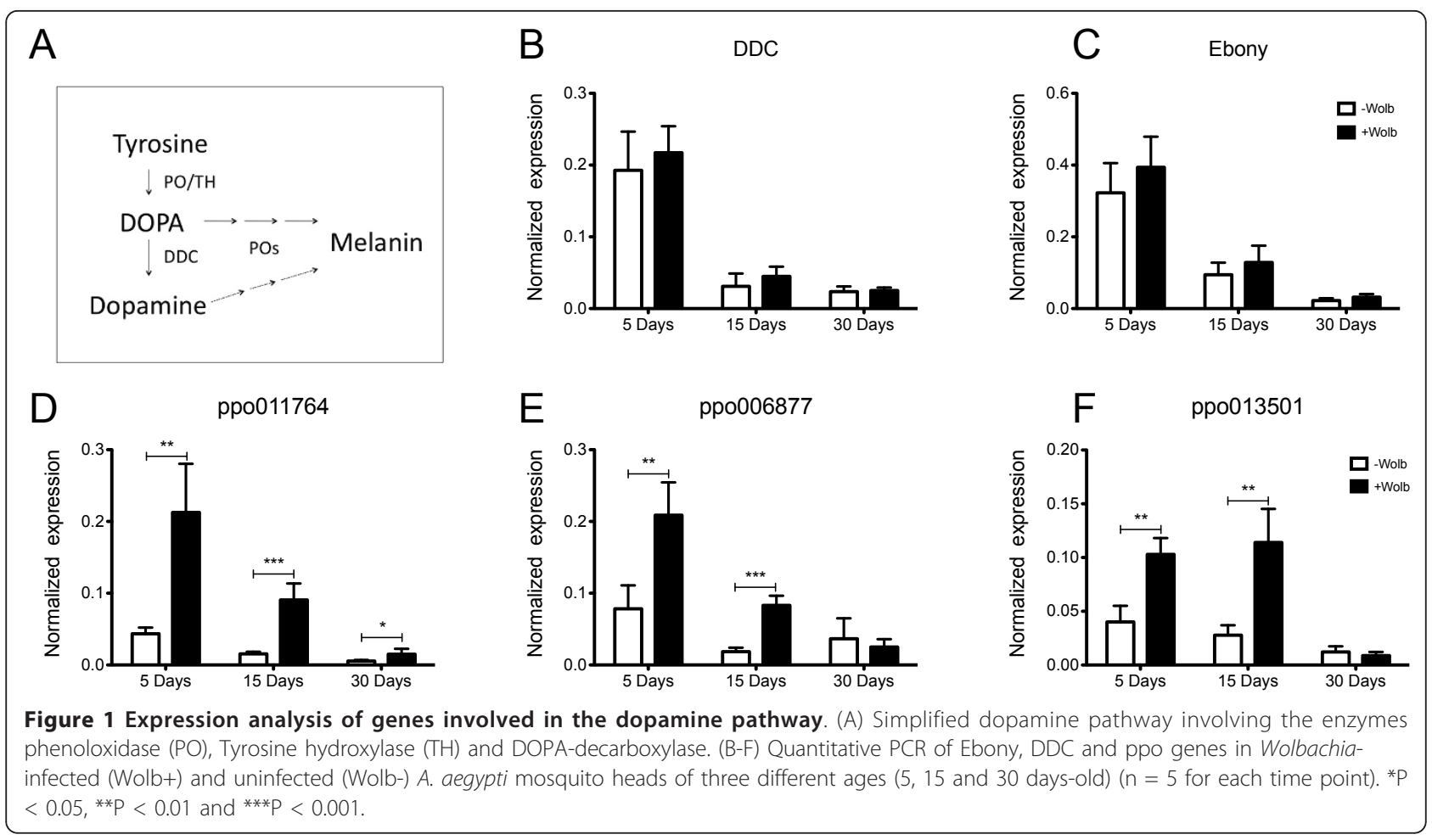

environment insectary at $26^{\circ} \mathrm{C}$ and approx. $80 \%$ humidity. All experiments were carried out on sucrose fed individuals. Thirty day-old Wolbachia-infected females were sorted into two different phenotypic categories normal and abnormal (containing "shaky" or "bendy") by placing insects into a cage and observing their probing behavior on a human hand [7].

For gene expression studies pools containing 20 female mosquitoes were dissected in ice-cold PBS, pooled, snap frozen in liquid nitrogen and extracted for total RNA using Trizol (Invitrogen Corp., Carlsbad, CA). Total RNA was treated with $2 \mu \mathrm{L}$ of DNase I (Roche) for 30 minutes at $37^{\circ} \mathrm{C}$ in a $20 \mu \mathrm{L}$ reaction to eliminate genomic DNA. Approximately $0.5 \mu \mathrm{g}$ of total RNA was reverse transcribed using random primers and SuperScript III reverse transcriptase (Invitrogen) according to manufacturer's protocols.

Quantitative real-time PCR (qRT-PCR) was used to measure the relative expression of a set of target genes that have been shown to be involved in dopamine synthesis and elimination (Figure 1A; Table 1). A. aegypti ribosomal protein $S 17$ (Ae-RpS17, Table 1) was used as a reference gene [22] and the single copy Wolbachia ankyrin repeat gene (WD0550) (ANK550, Table 1) was used to calculate the density of bacteria in individual mosquitoes when normalized to the S17 gene. RTqPCR was performed on a Rotor-gene 6000 (Corbett Life Science, Sydney, NSW) using Platinum ${ }^{\circledR}$ SYBR $^{\circledR}$ Green (Invitrogen Inc, Carlsbad, CA) according to the manufacturer's instructions. For each sample a mastermix of $2 \mu \mathrm{L}$ RNase-free water, $5 \mu \mathrm{L}$ of SYBR Supermix and $0.5 \mu \mathrm{L}$ of each primer $(5 \mu \mathrm{M})$ were added to $2 \mu \mathrm{L}$ of cDNA or gDNA. Three technical replicates were run for each sample. The cycling protocol was as follows; 2 minutes at $50^{\circ} \mathrm{C}$ for the UDG incubation, 2 minutes at $95^{\circ} \mathrm{C}$ for $\mathrm{Taq}$ activation, 40 cycles of denaturation at $95^{\circ}$ $\mathrm{C}$ for $5 \mathrm{~s}$, annealing at $60^{\circ} \mathrm{C}$ for $5 \mathrm{~s}$ and extension and fluorescence acquisition at $72^{\circ} \mathrm{C}$ for $15 \mathrm{~s}$ and then a melt curve analysis from $68-95^{\circ} \mathrm{C}$ in $1^{\circ} \mathrm{C}$ increments. The mean Cycle Threshold (CT) and mean amplification efficiency (E) per biological replicate was calculated from the three technical replicates using Rotor-Gene 6000 Series Software ver.1.7.75 (Info-ZIP Pty Ltd.). The raw output $\mathrm{CT}$ data was normalized by taking into consideration the differences in amplification efficiency of target and the reference genes using Q-gene software [23]. The data were then analysed using general linear models with age $(5,15$ or 30 days) and line (+Wolb or -Wolb) as factors using Statistica 8.0 (StatSoft, Inc.).

Expression of all genes reduces with age, regardless of infection status (Figure 1; see Table S1; Additional file 1). However expression is significantly higher in Wolbachia infected mosquitoes for the three-prophenoloxidase (ppo) genes, but not for DDC or Ebony (Figure 1B-F). The increased expression of genes related to the melanization pathway in Wolbachia infected insects is consistent with the finding that several immunity genes (including AAEL011764) are also up-regulated by the 
Table 1 Primers used for quantitative PCR analysis of genes related to dopamine metabolism

\begin{tabular}{|c|c|c|c|}
\hline Accession & Primer & Sequence $5^{\prime}-3^{\prime}$ & Amplicon (bp) \\
\hline \multirow[t]{2}{*}{ AAEL004175-RA } & Ae-RpS17F & CACTCCCAGGTCCGTGGTAT & 81 \\
\hline & Ae-RpS17R & GGACACTTCCGGCACGTAGT & \\
\hline \multirow[t]{2}{*}{ AAEL014238-RB } & $D d c F$ & GGTGGACTACATCGCCAACT & 166 \\
\hline & Ddc R & GACACCAGGCATGATGACAC & \\
\hline \multirow[t]{2}{*}{ AAEL005793-RA } & Ebony F & GAATCGGGACGGAGATTACA & 198 \\
\hline & Ebony R & ACGGCCACATITTCGTAGTC & \\
\hline \multirow[t]{2}{*}{ AAEL011764-RA } & Ppo011764 F & CTGAACAACGGATTCCCATT & 165 \\
\hline & Ppo011764R & TCATCAACGTCTGCACATCA & \\
\hline \multirow[t]{2}{*}{ AAEL006877-RA } & Ppo006877 F & CTATACGGCTTCGGATCGAG & 184 \\
\hline & Ppo006877 R & GTACCGAGCCATCGTTTGTT & \\
\hline \multirow[t]{2}{*}{ AAEL013501-RA } & Ppo013501 F & CTTCGGATCGAGAGCTTGAG & 176 \\
\hline & Ppo013501 R & GTACCGAGCCATCGTTTGTT & \\
\hline \multirow[t]{2}{*}{ NC_002978: C537094-538083 } & ANK550 F & CAGGAGTTGCTGTGGGTATATTAG & 74 \\
\hline & ANK550 R & TGCAGGTAATGCAGTAGCGTAAA & \\
\hline
\end{tabular}

presence of this bacterium in $A$. aegypti [3,4] and more recently that Wolbachia-infected mosquitoes exhibit higher levels of melanization in their hemolymph [24].

Dopamine levels were also determined in mosquitoes of three ages $(5,15$, and 30 days). Our hypothesis was that Wolbachia-infected mosquitoes, would exhibit lower levels of dopamine, resembling the situation in Parkinson's Disease in humans [17]. Mosquitoes were anesthetized with $\mathrm{CO}_{2}$ and then decapitated. Preliminary analysis indicated pooling 5 heads was sufficient to achieve a robust dopamine signal and hence all reported sample sizes represent pools of 5 heads. Heads were snap frozen on dry ice and kept at $-80^{\circ} \mathrm{C}$ for up to two weeks. One hundred microliters of $0.1 \mathrm{M}$ perchloric acid containing $5 \mathrm{ng}$ deoxyepinephrine (as an internal standard) was added to each tube. Heads were sonicated using a probe setting of $60 \% \mathrm{amp} / 20 \mathrm{Khz}$ for $5 \mathrm{sec}$, followed by $10 \mathrm{sec}$ rest and another $5 \mathrm{sec}$ sonication (on ice). Tubes were centrifuged for $5 \mathrm{~min}$ at 13,000 rpm. Supernatant was filtered through a $4 \mathrm{~mm} / 0.22$ syringe filter (PM Separations, Australia) and injected into a HPLC system that consisted of a degasser, autosampler and an isocratic pump (Model 1100, Agilent Technologies, Inc. CA), a Sunfire C18 column, $4.6 \mathrm{~mm} \times 150$ mm, 5 um; (Waters Corporation, MA) and a Coulochem III (ESA Laboratories, Inc. MA) electrochemical detector. The mobile phase was a $12 \%$ acetonitrile/75 mM potassium dihydrogen phosphate buffer containing 1 $\mathrm{mM}$ EDTA and $1.4 \mathrm{mM}$ octane sulfonic acid adjusted to $\mathrm{pH} 4.13$ with phosphoric acid. Flow rate was $1.2 \mathrm{ml} /$ min. The conditioning cell (Model 5020, ESA Laboratories, Inc. MA) operated at $+350 \mathrm{mV}$ with the first and second electrode of the analytical cell (Model 5014B, ESA Laboratories, Inc. MA) potentials set to $-150 \mathrm{mV}$ and $+250 \mathrm{mV}$ respectively. Data were quantified by calculating peak-height ratios for dopamine relative to deoxyepinephrine, and calibrated using a standard curve. Data were then processed with Chemstation software (Rev B.01.03, Agilent Technologies, Inc. CA). Samples were corrected for dilution and expressed as pg/mosquito head. Dopamine levels were analysed using standard t-tests comparing the effect of line (+Wolb or -Wolb) at each of three adult ages (5,15 or 30 days) using Statistica 8.0 (StatSoft, Inc.). In each case comparisons were only made between mosquitoes collected on the same day and analysed in the same batch.

The levels of dopamine reported here (2.2 pMol/head) are in keeping with previously reported measures for female $A$. aegypti heads [25]. Dopamine levels were significantly higher in five and 30 day-old mosquitoes when Wolbachia is present (Figure 2A,C). As we have previously seen abnormal phenotypes in older mosquitoes (> 15 days-old) we further analyzed dopamine contents in normal and Wolbachia-infected mosquitoes that exhibited these phenotypes (shaky or bendy). Results show that there was no difference between normal and abnormal mosquitoes (Figure 3A), although Wolbachia densities were higher in abnormal mosquitoes (Figure 3B). We can conclude then that dopamine levels alone cannot explain the phenotypes. Artificial manipulation of 


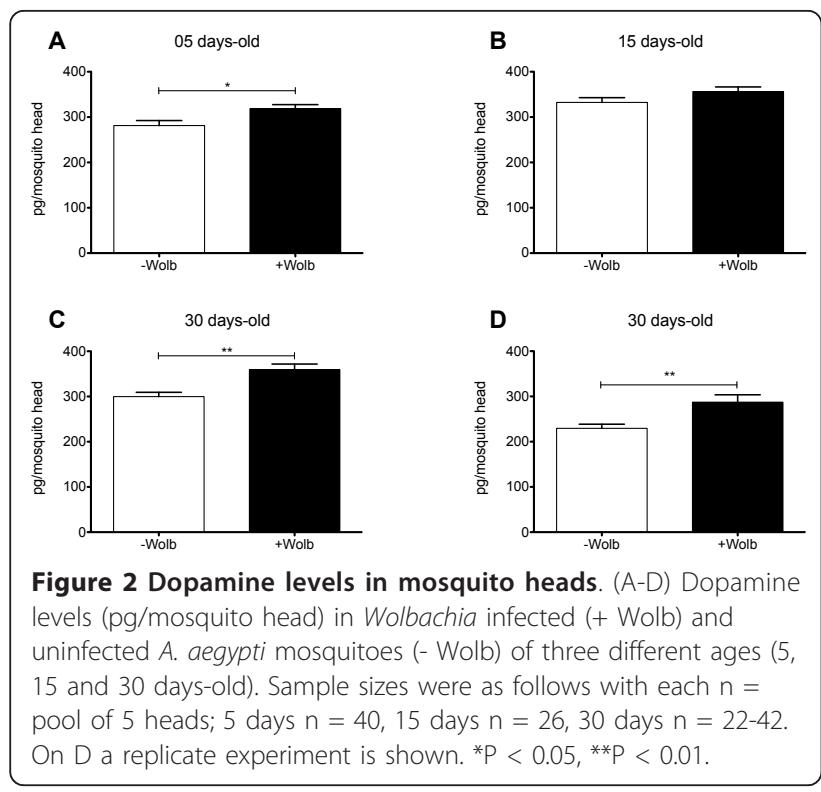

dopamine levels by chemical means in $A$. aegypti could be employed in future studies in an attempt to determine what if any effect the Wolbachia-induced elevation in dopamine is having on the biology of the insect.

Taken together, our findings indicate that Wolbachiainfected mosquitoes have higher dopamine levels in their heads, although the effect was not present at all ages examined. These higher dopamine levels are consistent with previous observation that Wolbachiainfected mosquitoes exhibit higher activity [6]. There may be other behaviours governed by dopamine that are also affected by Wolbachia infection however there is no obvious correlation between higher dopamine levels found here and the abnormal behavioral phenotypes "shaky" and "bendy" we examined. The positive correlation between Wolbachia densities and prevalence of behavioral phenotypes suggests that bacterial densities are predictive of virulence.

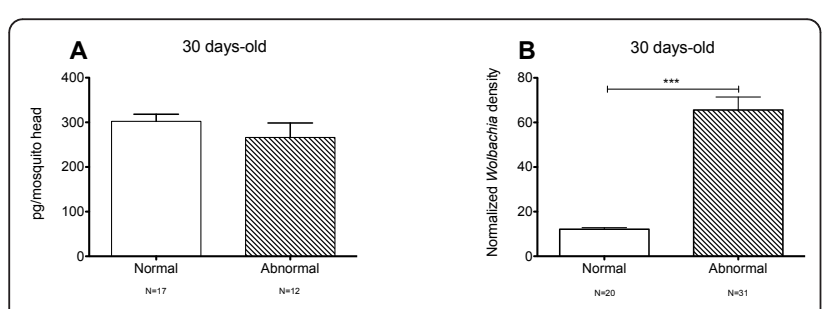

Figure 3 Dopamine levels in phenotyped mosquitoes. (A) Dopamine levels in 30 days-old Wolbachia-positive mosquitoes exhibiting normal or abnormal behavior ("shaky" or "bendy", see text) ( $n=$ number of pools). (B) Normalized Wolbachia density (ankirin gene: RPS17, see text) in individual mosquitoes exhibiting normal or abnormal behavior ( $\mathrm{n}=$ number of individual females). ***P $<0.0001$

\section{Additional material}

Additional file 1: Statistical analysis of gene expression. Table S1 shows the analysis of quantitative expression of mosquito genes related to dopamine pathway

\section{Acknowledgements}

We are grateful to Nichola Kenny and Jenny Gough for assistance with mosquito rearing. This research was supported by a grant from the Foundation for the National Institutes of Health through the Grand Challenges in Global Health Initiative of the Bill and Melinda Gates Foundation and the National Health and Medical Research Council, Australia.

\section{Author details}

${ }^{1}$ School of Biological Sciences, The University of Queensland, Brisbane Qld 4072, Australia. ${ }^{2}$ René Rachou Research Institute- FIOCRUZ, Belo Horizonte MG 30190, Brazil. ${ }^{3}$ Queensland Brain Institute, The University of Queensland, Brisbane Qld 4072, Australia.

\section{Authors' contributions}

LAM conceived the studied, helped on the dopamine assays and drafted the manuscript. YE carried out the gene expression studies. KT performed the dopamine assays. DE participated in the design of the study and helped to draft the manuscript. EAM helped on statistical analysis and to draft the manuscript. SLO conceived of the study, participated in its design and coordination and helped to draft the manuscript. All authors read and approved the final manuscript.

\section{Competing interests}

The authors declare that they have no competing interests.

Received: 22 November 2010 Accepted: 28 February 2011

Published: 28 February 2011

\section{References}

1. WHO: Dengue and Dengue Hemorragic Fever. In Book Dengue and Dengue Hemorragic Fever. Volume 117. City: WHO; 2009, (Editor ed.^eds.).

2. McMeniman CJ, Lane RV, Cass BN, Fong AW, Sidhu M, Wang YF, O'Neill SL: Stable introduction of a life-shortening Wolbachia infection into the mosquito Aedes aegypti. Science 2009, 323:141-144.

3. Kambris Z, Cook PE, Phuc HK, Sinkins SP: Immune activation by lifeshortening Wolbachia and reduced filarial competence in mosquitoes. science 2009, 326:134-136.

4. Moreira LA, Iturbe-Ormaetxe I, Jeffery JA, Lu G, Pyke AT, Hedges LM, Rocha BC, Hall-Mendelin S, Day A, Riegler M, et al: A Wolbachia symbiont in Aedes aegypti limits infection with dengue, Chikungunya, and Plasmodium. Cell 2009, 139:1268-1278.

5. Bian G, Xu Y, Lu P, Xie Y, Xi Z: The endosymbiotic bacterium Wolbachia induces resistance to dengue virus in Aedes aegypti. PLoS Pathog 2010, 6:e1000833.

6. Evans O, Caragata EP, McMeniman CJ, Woolfit M, Green DC, Williams CR, Franklin CE, O'Neill SL, McGraw EA: Increased locomotor activity and metabolism of Aedes aegypti infected with a life-shortening strain of Wolbachia pipientis. J Exp Biol 2009, 212:1436-1441.

7. Moreira LA, Saig E, Turley AP, Ribeiro JM, O'Neill SL, McGraw EA: Human probing behavior of Aedes aegypti when infected with a life-shortening strain of Wolbachia. PLoS Negl Trop Dis 2009, 3:e568

8. Turley AP, Moreira LA, O'Neill SL, McGraw EA: Wolbachia infection reduces blood-feeding success in the dengue fever mosquito, Aedes aegypti. PLoS Negl Trop Dis 2009, 3:e516.

9. Neckameyer WS, Leal SM: Biogenic Amines as Circulating Hormones in Insects. In Hormones, Brain and behavior. Volume 3. Edited by: Pfaff DW, Arnold AP, Etgen AM, Fahrbach SE, Rubin RT. San Diego: Academic Press; 2002:141-165.

10. Davis MM, Primrose DA, Hodgetts RB: A member of the p38 mitogenactivated protein kinase family is responsible for transcriptional induction of Dopa decarboxylase in the epidermis of Drosophila melanogaster during the innate immune response. Mol Cell Biol 2008, 28:4883-4895. 
11. Ferdig MT, Li J, Severson DW, Christensen BM: Mosquito dopa decarboxylase CDNA characterization and blood-meal-induced ovarian expression. Insect Mol Biol 1996, 5:119-126.

12. Gruntenko NE, Rauschenbach IY: Interplay of JH, 20E and biogenic amines under normal and stress conditions and its effect on reproduction. $J$ Insect Physiol 2008, 54:902-908.

13. Huang CY, Chou SY, Bartholomay LC, Christensen BM, Chen CC: The use of gene silencing to study the role of dopa decarboxylase in mosquito melanization reactions. Insect Mol Biol 2005, 14:237-244.

14. Christensen BM, Li J, Chen CC, Nappi AJ: Melanization immune responses in mosquito vectors. Trends Parasitol 2005, 21:192-199.

15. Wicker-Thomas C, Hamann M: Interaction of dopamine, female pheromones, locomotion and sex behavior in Drosophila melanogaster. $J$ Insect Physiol 2008, 54:1423-1431.

16. Baik JH, Picetti R, Saiardi A, Thiriet G, Dierich A, Depaulis A, Le Meur M, Borrelli E: Parkinsonian-like locomotor impairment in mice lacking dopamine D2 receptors. Nature 1995, 377:424-428.

17. Elsworth JD, Roth RH: Dopamine synthesis, uptake, metabolism, and receptors: relevance to gene therapy of Parkinson's disease. Exp Neurol 1997, 144:4-9.

18. Nagatsu T, Levitt M, Udenfriend S: Tyrosine Hydroxylase: THE INITIAL STEP IN NOREPINEPHRINE BIOSYNTHESIS. J Biol Chem 1964, 239:2910-2917.

19. Suh J, Jackson FR: Drosophila Ebony Activity Is Required in Glia for the Circadian Regulation of Locomotor Activity. Neuron 2007, 55:435-447.

20. Borycz J, Borycz JA, Loubani M, Meinertzhagen IA: tan and ebony genes regulate a novel pathway for transmitter metabolism at fly photoreceptor terminals. J Neurosci 2002, 22:10549-10557.

21. Kanehisa M, Goto S: KEGG: kyoto encyclopedia of genes and genomes. Nucleic Acids Res 2000, 28:27-30

22. Cook PE, Hugo LE, Iturbe-Ormaetxe I, Williams CR, Chenoweth SF, Ritchie SA, Ryan PA, Kay BH, Blows MW, O'Neill SL: The use of transcriptional profiles to predict adult mosquito age under field conditions. Proc Natl Acad Sci USA 2006, 103:18060-18065.

23. Simon P: Q-Gene: processing quantitative real-time RT-PCR data. Bioinformatics 2003, 19:1439-1440.

24. Thomas P, Kenny N, Eyles D, Moreira LA, O'Neil SL, Asgari S: Infection with the wMel and wMelPop strains of Wolbachia leads to higher levels of melanization in the hemolymph of Drosophila melanogaster, Drosophila simulans and Aedes aegypti. Dev Comp Immunol 2011.

25. Andersen JP, Schwartz A, Gramsbergen JB, Loeschcke V: Dopamine levels in the mosquito Aedes aegypti during adult development, following blood feeding and in response to heat stress. J Insect Physiol 2006, 52:1163-1170.

doi:10.1186/1756-3305-4-28

Cite this article as: Moreira et al:: The wMelPop strain of Wolbachia interferes with dopamine levels in Aedes aegypti. Parasites \& Vectors 2011 $4: 28$

\section{Submit your next manuscript to BioMed Central and take full advantage of:}

- Convenient online submission

- Thorough peer review

- No space constraints or color figure charges

- Immediate publication on acceptance

- Inclusion in PubMed, CAS, Scopus and Google Scholar

- Research which is freely available for redistribution 